\title{
Robust Stability in Discrete Control Systems via Linear Controllers with Single and Delayed Time
}

\author{
B. L. Hernández-Galván, ${ }^{1}$ J. A. López-Rentería $(\mathbb{D}){ }^{1,2}$ \\ B. Aguirre-Hernández, ${ }^{3}$ and G. Fernández-Anaya ${ }^{1}$ \\ ${ }^{1}$ Departamento de Física y Matemáticas, Universidad Iberoamericana, CP 01219, CDMX, Mexico \\ ${ }^{2}$ Departamento de Ingeniería Eléctrica y Electrónica, TNM-Instituto Tecnológico de Tijuana-CONACYT, CP 22414, \\ Tijuana, BC, Mexico \\ ${ }^{3}$ Departamento de Matemáticas, Universidad Autónoma Metropolitana, CP 09340, CDMX, Mexico
}

Correspondence should be addressed to J. A. López-Rentería; jorge.lopez@tectijuana.edu.mx

Received 29 November 2017; Revised 16 May 2018; Accepted 4 June 2018; Published 27 June 2018

Academic Editor: Luis J. Yebra

Copyright (C) 2018 B. L. Hernández-Galván et al. This is an open access article distributed under the Creative Commons Attribution License, which permits unrestricted use, distribution, and reproduction in any medium, provided the original work is properly cited.

In this work, a discrete feedback of single and a delayed time is introduced in a LTI control discrete system, yielding a monoparametric family of LTI systems. A polynomial approach technique to compute the maximal robust stability interval of the monoparametric system with single and delayed time controller is developed by using the zero exclusion principle and the boundary crossing theorem. Illustrative examples are given to show the technique.

\section{Introduction}

Discrete LTI control systems have been object of study and design in the last years due to the great quantity of applications as in digital devices as well in mechanical plants and others. Particularly, the discrete-time control systems of interest are the so-called SISO (single input-single output), which are LTI (linear time invariant [1-4]). It is well known that the internal evolution of the system is determined by its poles (also referred to as the eigenvalues of its associated matrix in state space representation) which can be computed by finding the roots (zeroes or solutions) of its characteristic polynomial. One of the most worked problems concerning the dynamics of a system is the stabilization problem. Namely, a LTI discrete system is BIBO stable (bounded input-bounded output) if and only if its transfer function has no poles with modulus greater than or equal to one. That is, the poles of the system must lie inside the open unit complex disk. This class of stability for discrete systems is also called Schur stability ([5-8]). In this sense, a linear discrete-time invariant system is said to be stable if and only if its characteristic polynomial is a Schur polynomial. A polynomial is said to be stable if its roots lie in the stability zone (in our case of study, the stability zone is the open unit complex disk for Schur stability). There exists a lot of criteria to determinate the Schur stability of a polynomial, as the Schur-Cohn and Jury's criterion [9-12]. In [13, 14], modified Schur-Cohn and Jury's criterion are given. With respect to polynomial families in topological sense, sufficient conditions to determine an interval of Schur polynomials are given in [15-17]; techniques to locate the roots inside the unit circle of a polytope of polynomials are developed; moreover, a robust Hurwitz stability criterion for polynomial intervals is proposed in [18] based on Kharitonov rectangles and in [19] set values are given for polynomials Schur stable, while a method to study the Schur stability of a segment of polynomials was reported recently in [20]. Concerning rays of polynomials, the case of finding the maximal Hurwitz stability (roots with negative real part) interval was solved by Bialas in 1985 [21]. An improved technique to determinate the Hurwitz stability of the ray was given in [8], and these results are used in [22] in order to generate chaos. Good references where the stability of families of polynomials can be consulted are in the books [5-7]. 
A related problem is the inclusion of discrete controllers with single delays; for instance, in [23] a technique to determinate an interval of Schur stability for sample-data systems with a delayed control is given and in [24] Schur stability in linear discrete-time systems with single-delay using the method of Lyapunov functions is studied. In the following work, we shall provide a technique that ensure the Schur stability of a ray of polynomials, which is the characteristic family of polynomials of a linear discrete-time system in closed-loop with a feedback linear control in both single and delayed time.

The rest of the paper is organized as follows: In Section 3, the description of the system is given as well as the problems statement. In Section 4, preliminary tool to solve the proposed problems is introduced. The main results are provided in Section 5 which is divided into three subsections: In Section 5.1, generalizations of the boundary theorems in its discrete version are shown; in Section 5.2, the obtained generalizations are applied on a technique to find the maximal robust Schur stability interval of the uncertainty present on a polynomial ray, and, therefore, robust stability of a discrete LTI system in closed-loop with a linear controller. An illustrative example that shows the technique is also given. Finally, in Section 5.3 the technique is extended to discrete control systems with a controller in single time-delay and applied to an example.

\section{Basic Notations}

$\mathbb{N}$ : set of natural numbers

$\mathbb{R}$ : set of real numbers

$\mathbb{R}^{n}$ : set of $n$-tuples of real numbers

$\mathbb{R}^{n \times n}$ : set of real square matrices of size $n \times n$

$\mathbb{C}$ : set of complex numbers

$\mathbb{C}^{-}$: set of complex numbers with negative real part

$\mathbb{C}^{+}$: set of complex numbers with positive real part

$i \mathbb{R}$ : imaginary pure complex numbers-complex axis

D: open unit complex disk-complex numbers with modulus less than 1

$\partial A$ : boundary of the set $A$

$\bar{A}$ : closure of the set $A$

$A-B$ : difference between sets-set $A$ without elements in common with $B$

$\sigma(f)$ : set of zeroes (roots) of the function (polynomial) $f$

$\operatorname{det}(A)$ : determinant of a square matrix $A$.

\section{Problems Statement}

Consider the discrete LTI control system

$$
x_{m+1}=A x_{m}+b u_{m},
$$

where $x_{m} \in \mathbb{R}^{n}$ is the states variable vector, $A \in \mathbb{R}^{n \times n}$ and $b \in \mathbb{R}^{n}$ are given in controllable canonical form (also called Jerk realization) as

$$
A=\left[\begin{array}{ccccc}
0 & 1 & 0 & \cdots & 0 \\
0 & 0 & 1 & \cdots & 0 \\
\vdots & \vdots & \vdots & \ddots & \vdots \\
0 & 0 & 0 & \cdots & 1 \\
-a_{n} & -a_{n-1} & -a_{n-2} & \cdots & -a_{1}
\end{array}\right] \text {, }
$$

$$
b=\left[\begin{array}{c}
0 \\
0 \\
\vdots \\
1
\end{array}\right] \text {, }
$$

and $u_{m} \in \mathbb{R}$ is the linear actuator of the system. The openloop characteristic polynomial is $p_{A}(z)=z^{n}+a_{1} z^{n-1}+\cdots+$ $a_{n-1} z+a_{n}$, where $z$ is the complex $z$-transform variable.

Case 1. For system (1) with feedback control $u_{m}=-k c^{T} x_{m}$, with $c^{T}=\left(c_{n}, \ldots, c_{1}\right)$, the closed-loop characteristic polynomial is the ray of polynomials $P(z, k)=p_{A}(z)+k p_{c}(z)$, where $p_{c}(z)=c_{1} z^{n-1}+c_{2} z^{n-2}+\cdots+c_{n-1} z+c_{n}$.

Case 2. For system (1) with the feedback control $u_{m-\tau}=$ $-k c^{T} x_{m-\tau}$, where $\tau \in \mathbb{N}$ and $c^{T}=\left(c_{n}, \ldots, c_{1}\right)$, the closed-loop characteristic polynomial is the ray of polynomials $P_{\tau}(z, k)=$ $\tilde{p}_{A}(z)+k \widetilde{p}_{c}(z)$ (further details about this characteristic polynomial will be given in Section 5.3).

If we suppose that $p_{A}(z)$ is a Schur polynomial, then we can to perturb the parameter $k$ of the controller (for both of the Cases 1 and 2) to keep stability. Then, the objective is to compute the maximal perturbation of $k$, that is, the minimum $k<0$ and the maximum $k>0$, to obtain $k_{\min }^{-}$and $k_{\max }^{+}$, respectively, such that system (1) has poles inside the unit complex disk $\mathscr{D}$ for all $k$ in the maximal stability interval $K=\left(k_{\min }^{-}, k_{\max }^{+}\right)$. In other words, the system is robustly Schur stable in the maximal stability interval $K$. Any other subset $[a, b] \subset K$ is just an interval of robust stability. The technique will be based on the boundary theorems: the zero exclusion principle (ZEP) and the boundary crossing theorem (BCT).

\section{Preliminaries}

4.1. The Boundary Theorems Generalized. Let us consider the complex plane $\mathbb{C}$ and let $\mathcal{S} \subset \mathbb{C}$ be any given open set. We know that $\mathcal{S}$, its boundary $\partial \mathcal{S}$, and the complement $\mathcal{U}=$ $\mathbb{C}-\mathcal{S}$ form a disjoint partition of the complex plane. In the following we give the tool used in [8] to find the maximal Hurwitz stability interval for a ray of real polynomials. The discrete versions are given in the following section of main results.

The classical boundary theorems consider the following hypothesis. 
Hypothesis 1. Consider the polynomial family

$$
\begin{aligned}
P(\lambda, k)= & a_{0}(k) \lambda^{n}+a_{1}(k) \lambda^{n-1}+\cdots+a_{n-1}(k) \lambda \\
& +a_{n}(k),
\end{aligned}
$$

in the variable $\lambda \in \mathbb{C}$ and uncertainty parameter $k \in \mathbb{R}$, satisfying the following:

(i) having fixed degree $n$,

(ii) with continuous coefficients with respect to $k$ on a fixed interval $I=[a, b]$.

The generalizations of the boundary theorems used in [8] also use the same hypothesis for the roots criterion by taking $\mathcal{S}=$ $\mathbb{C}^{-}$which is the Hurwitz stability zone. In the following we shall give a version of them.

Theorem 1 (generalization of BCT). Under Hypothesis 1, suppose that $P(\lambda, a)$ has $n_{1}$ roots in $\mathbb{C}^{-}$and $n-n_{1}$ roots in $\mathbb{C}^{+}$, and $P(\lambda, b)$ has $m_{1}$ roots in $\mathbb{C}^{-}$and $n-m_{1}$ roots in $\mathbb{C}^{+}$. If $n_{1} \neq m_{1}$, then there exists at least one $\rho$ in $(a, b]$ such that

(i) $P(\lambda, \rho)$ has at least $n_{1}$ roots in $\mathbb{C}^{-} \cup i \mathbb{R}$,

(ii) $P(\lambda, \rho)$ has at least $n-n_{1}$ roots in $\mathbb{C}^{+} \cup i \mathbb{R}$,

(iii) $P(\lambda, \rho)$ has at least one root in $i \mathbb{R}$.

Similarly, under the same hypothesis and with the same stability zone $\mathcal{S}=\mathbb{C}^{-}$, the generalization of the zero exclusion principle is given as follows.

Theorem 2 (generalization of ZEP). Consider the polynomial family $P(\lambda, k)$ under Hypothesis 1. Suppose that for some $\rho \in$ $(a, b)$ the family has $n_{1}$ roots in $\mathbb{C}^{-}$and $n-n_{1}$ roots in $\mathbb{C}^{+}$. Then the entire family keeps $n_{1}$ roots in $\mathbb{C}^{-}$and $n-n_{1}$ roots in $\mathbb{C}^{+}$if and only if

$$
P(i \omega, k) \neq 0 \quad \forall k \in[a, b] \text { and } \forall \omega \in \mathbb{R} .
$$

An important result that is used to prove the previous generalized theorems is the continuous dependence of roots (CDR), which establishes the preservation of continuity between roots and coefficients of a polynomial family.

Lemma 3 (CDR, [5]). Consider the monoparametric family of polynomials $P(\lambda, k)=\sum_{j=0}^{n} a_{j}(k) \lambda^{n-j}$ under Hypothesis 1 . Then the roots of $P(\lambda, k)$ vary continuously with respect to $k \in$ $[a, b]$. That is, there exist continuous mappings $\lambda_{j}:[a, b] \rightarrow \mathbb{C}$ for $j=1,2, \ldots, n$ such that $\lambda_{1}(k), \ldots, \lambda_{n}(k)$ are the roots of $P(\lambda, k)$.

\section{Main Results}

We have divided this section in three parts. In the Section 5.1 we present adaptation of the before generalizations theorems to discrete case. In Section 5.2, boundary theorems based technique for calculating the maximal Schur stability interval for a discrete family of systems using linear feedback with single time is developed. And in Section 5.3 the technique is extended to a single time-delay controller.
5.1. The Discrete Generalized Boundary Theorems. It is not too hard to see that the generalizations of the boundary theorems can be easily adaptable in the case of $\mathcal{S}=\mathscr{D}$ instead of $\mathbb{C}^{-}$. However, it is necessary to present the discrete version of these generalizations in order to apply them in the aforementioned technique.

Remark 4. The polynomials to consider come from a real discrete control system whose characteristic polynomial comes from the $z$-transform applied to the discrete system. Thus, all of the polynomials to deal with in the coming development have real coefficients and complex variable $z$ instead of $\lambda$.

Theorem 5 (discrete BCT generalized). Consider the polynomial family $P(z, k)$ on the complex variable $z$ under Hypothesis 1. Suppose that $P(z, a)$ has $n_{1}$ roots in $\mathscr{D}$ and $n-n_{1}$ roots in $\mathbb{C}-\overline{\mathscr{D}}$; and $P(z, b)$ has $m_{1}$ roots in $\mathscr{D}$ and $n-m_{1}$ roots in $\mathbb{C}-\overline{\mathscr{D}}$. Then, if $n_{1} \neq m_{1}$ there exist at least one $\rho$ in $(a, b]$ such that

(i) $P(z, \rho)$ has at least $n_{1}$ roots in $\overline{\mathscr{D}}$,

(ii) $P(z, \rho)$ has at least $n-n_{1}$ roots in $\mathbb{C}-\overline{\mathscr{D}}$,

(iii) $P(z, \rho)$ has at least one root in $\partial \mathscr{D}$.

Proof. Since $P(z, k)$ satisfies Hypothesis 1 , then by Lemma 3 there exist $n$ continuous function roots of $P(z, k)$, say $z_{1}(k), \ldots, z_{n}(k) \in \mathbb{C}$. Without loss of generality we can suppose that, for $j=1, \ldots, n_{1}$, we have that $z_{j}(a) \in \mathscr{D}$ and, for $j=n_{1}+1, \ldots, n$, we have that $z_{j}(a) \in \mathbb{C}-\overline{\mathscr{D}}$. Since $n_{1} \neq m_{1}$, by continuity at least one root traveled through the complex plane collapsing into $\partial \mathscr{D}$ for some $\rho \in(a, b]$, resulting in the items claimed.

Similarly, the generalized zero exclusion principle can be adapted to the discrete case in the following way.

Theorem 6 (generalization of ZEP). Consider the polynomial family $P(z, k)$ under Hypothesis 1. Suppose that for some $\rho \in$ $(a, b)$ the family has $n_{1}$ roots in $\mathscr{D}$ and $n-n_{1}$ roots in $\mathbb{C}-\overline{\mathscr{D}}$. Then, the entire family keeps $n_{1}$ roots in $\mathscr{D}$ and $n-n_{1}$ roots in $\mathbb{C}-\overline{\mathscr{D}}$ if and only if

$$
P\left(e^{i \theta}, k\right) \neq 0 \quad \forall k \in[a, b] \text { and } \forall \theta \in[0,2 \pi] .
$$

Proof. $(\Rightarrow)$ If all of the elements of the family have $n_{1}$ roots in $\mathscr{D}$ and $n-n_{1}$ roots in $\mathbb{C}-\overline{\mathscr{D}}$, then it is clear that there are no roots in the unit circumference $\partial \mathscr{D}$. Therefore, $P\left(e^{i \theta}, k\right) \neq 0$, for all $0 \leq \theta \leq 2 \pi$ and for all $k \in[a, b] . \quad(\Leftarrow)$ Suppose that $P\left(e^{i \theta}, k\right) \neq 0$ for all $0 \leq \theta \leq 2 \pi$ and for all $k \in[a, b]$. Let us proceed by contradiction. Suppose that there is $k_{0} \in(a, b]$ such that the polynomial $P\left(z, k_{0}\right)$ has $m_{1}$ roots in $\mathscr{D}$ and $n-m_{1}$ roots in $\mathbb{C}-\overline{\mathscr{D}}$ with $n_{1} \neq m_{1}$. Then, from Theorem 5 , there exists a $\rho \in(a, b]$ such that $P\left(e^{i \theta_{0}}, \rho\right)=0$ for some $0 \leq \theta_{0} \leq$ $2 \pi$, which is a contradiction.

5.2. The Roots Criterion for Discrete-Time Linear Systems. Next, we shall develop the technique to compute the maximal Schur stability interval. Let $p_{A}(z)=z^{n}+a_{1} z^{n-1}+\cdots+a_{n}$ 
be an $n$-fixed degree polynomial and $p_{c}(z)=c_{1} z^{m}+\cdots+$ $c_{m} z+c_{m+1}$ be a polynomial such that $n>m$. We desire to find the values of $k$ around $k=0$ such that the ray $P(z, k)=$ $p_{A}(z)+k p_{c}(z)$ has $n$ roots inside $\mathscr{D}$. Since $p_{A}(z)$ is a Schur polynomial, then $p_{A}\left(z^{-1}\right)$ has $n$ roots outside $\mathscr{D}$. Thus, the discrete generalized zero exclusion principle will be applied to the family $\mathscr{P}(z, k)=p_{A}\left(z^{-1}\right) P(z, k)$, which has $n$ roots inside $\mathscr{D}$ and $n$ roots outside $\mathscr{D}$ for $k=0$. The idea is to extend $(a, b)$ to the maximum interval for $k$ where the zero exclusion principle is satisfied and, therefore, we obtain robust stability. Firstly, let us carry $\left.\mathscr{P}(z, k)\right|_{z=e^{i \theta}}$ in a suitable form. get

By evaluating $p_{A}(z)$ and $p_{c}(z)$ in $z=e^{i \theta}, 0 \leq \theta \leq 2 \pi$ we

$$
\begin{aligned}
& p_{A}\left(e^{i \theta}\right)=R(\theta)+i Q(\theta) \\
& p_{c}\left(e^{i \theta}\right)=r(\theta)+i q(\theta)
\end{aligned}
$$

where

$$
\begin{aligned}
& R(\theta)=\cos (n \theta)+\sum_{j=1}^{n} a_{j} \cos [(n-j) \theta], \\
& Q(\theta)=\sin (n \theta)+\sum_{j=1}^{n-1} a_{j} \sin [(n-j) \theta], \\
& r(\theta)=\sum_{j=0}^{m} c_{j+1} \cos [(m-j) \theta], \\
& q(\theta)=\sum_{j=0}^{m-1} c_{j+1} \sin [(m-j) \theta] .
\end{aligned}
$$

Thus, the family $\left.P(z, k)\right|_{z=e^{i \theta}}$ can be written as

$$
\begin{aligned}
P\left(e^{i \theta}, k\right) & =p_{A}\left(e^{i \theta}\right)+k p_{c}\left(e^{i \theta}\right) \\
& =[R+k r](\theta)+i[Q+k q](\theta)
\end{aligned}
$$

Now, since $\left.p_{A}\left(z^{-1}\right)\right|_{z=e^{i \theta}}=p_{A}\left(e^{-i \theta}\right)=R(\theta)-i Q(\theta)$, the family $\mathscr{P}(z, k)$ evaluated in the unit circle becomes on

$$
\begin{aligned}
\mathscr{P}\left(e^{i \theta}, k\right)= & p_{A}\left(e^{-i \theta}\right) P\left(e^{i \theta}, k\right) \\
= & p_{A}\left(-e^{i \theta}\right) p_{A}\left(e^{i \theta}\right)+k p_{A}\left(e^{-i \theta}\right) p_{c}\left(e^{i \theta}\right) \\
= & {\left[R^{2}+Q^{2}\right](\theta)+k[R r+Q q](\theta) } \\
& +i k[R q-Q r](\theta) .
\end{aligned}
$$

Next, define the functions

$$
\begin{aligned}
& F(\theta)=[R r+Q q](\theta) ; \\
& G(\theta)=\left[R^{2}+Q^{2}\right](\theta) ; \\
& H(\theta)=[R q-Q r](\theta) .
\end{aligned}
$$

Thence, we can rewrite $\mathscr{P}\left(e^{i \theta}, k\right)$ as

$$
\mathscr{P}\left(e^{i \theta}, k\right)=G(\theta)+k F(\theta)+i k H(\theta),
$$

and this is the polynomial family form to be applied the zero exclusion principle in order to compute the maximal stability interval.

Remark 7. Some properties of $F, G$, and $H$ are as follows:

(i) $G(\theta) \neq 0$, for all $0 \leq \theta \leq 2 \pi$.

(ii) $H$ is an odd function and $H(0)=0, H(\pi)=0$.

(iii) $F$ is an even function.

Proof. Before anything, it is not hard to see that $Q(0)=q(0)=$ $0, Q(\pi)=q(\pi)=0, Q(-\theta)=-Q(\theta), q(-\theta)=-q(\theta), R(-\theta)=$ $R(\theta)$, and $r(-\theta)=r(\theta)$.

Proof of (i): $G(\theta)=\left[R^{2}+Q^{2}\right](\theta)=\left|p_{A}\left(e^{i \theta}\right)\right|^{2} \neq 0$, since $p_{A}\left(e^{i \theta}\right) \neq 0$, for all $0 \leq \theta \leq 2 \pi$, since $p_{A}(z)$ is Schur.

Proof of (ii): $H(0)=Q(0) r(0)-R(0) q(0)=0$ and similarly $H(\pi)=0$. Now, if $\theta \in[0,2 \pi]$, then

$$
\begin{aligned}
H(-\theta) & =Q(-\theta) r(-\theta)-R(-\theta) q(-\theta) \\
& =-Q(\theta) r(\theta)+R(\theta) q(\theta)=-H(\theta),
\end{aligned}
$$

and consequently, $H$ is an odd function.

Finally, proof of (iii):

$$
\begin{aligned}
F(-\theta) & =R(-\theta) r(-\theta)+Q(-\theta) q(-\theta) \\
& =R(\theta) r(\theta)+Q(\theta) q(\theta)=F(\theta),
\end{aligned}
$$

and, therefore, $F$ is an even function.

Item (ii) shows that $\theta=0, \pi$ are always zeroes of $H$ and if $\theta_{0} \in \sigma(H)$ then its conjugate $-\theta_{0}$ is also a zero of $H$. In the incoming development it will be shown a condition where $F(0) \neq 0$ in item (iii).

Let $\sigma(f)=\{\zeta \in \mathbb{C} \mid f(\zeta)=0\}$ denote the set of zeroes for a function $f(\zeta)$. In our case of studio we have the variable $0 \leq \theta \leq 2 \pi$; thus the set $\sigma(H)$ is the set of zeroes of $H$ modulo $2 \pi$. With the functions $F(\theta), G(\theta)$, and $H(\theta)$ defined above, let us define the sets

$$
\begin{aligned}
& \left.K^{+}=\left\{F\left(\theta_{l}\right) \mid \theta_{l} \in \sigma(H), F\left(\theta_{l}\right)>0\right)\right\} \\
& \left.K^{-}=\left\{F\left(\theta_{l}\right) \mid \theta_{l} \in \sigma(H), F\left(\theta_{l}\right)<0\right)\right\}
\end{aligned}
$$

If there are no elements in $\sigma(H)$ such that $F\left(\theta_{l}\right)>0$, then we will define $K^{+}=\left\{0^{+}\right\}$. Similarly, if there are no elements in $\sigma(H)$ such that $F\left(\theta_{l}\right)<0$, then we will define $K^{-}=\left\{0^{-}\right\}$.

Finally, the method to compute the maximal robust Schur stability interval is derived in the following result.

Theorem 8. Let $p_{A}(z)=z^{n}+a_{1} z^{n-1}+\cdots+a_{n}$ be a real Schur polynomial and consider any real polynomial $p_{c}(z)=c_{1} z^{m}+$ $\cdots+c_{m} z+c_{m+1}$, with $n>m$. Then, the maximal Schur stability interval for the monoparametric polynomial family $P(z, k)=$ $p_{A}(z)+k p_{c}(z)$ is $\left(k_{\min }^{-}, k_{\max }^{+}\right)$, where

$$
\begin{aligned}
& k_{\min }^{-}=\max \left\{-\frac{G\left(\theta_{l}\right)}{F\left(\theta_{l}\right)} \mid F\left(\theta_{l}\right) \in K^{+}\right\} \\
& k_{\max }^{+}=\min \left\{-\frac{G\left(\theta_{l}\right)}{F\left(\theta_{l}\right)} \mid F\left(\theta_{l}\right) \in K^{-}\right\} .
\end{aligned}
$$


Proof. The proof will be based on the discrete generalized zero exclusion principle applied to $\mathscr{P}(z, k)=p_{A}\left(z^{-1}\right) P(z, k)$; since $k=0$, the polynomial $\mathscr{P}(z, 0)=p_{A}\left(z^{-1}\right) p_{A}(z)$ has $n$ roots in $\mathscr{D}$ and $n$ roots in $\mathbb{C}-\overline{\mathscr{D}}$. That is, we have a member of the family $\mathscr{P}(z, k)$ with $n$ roots inside the open unit disk and $n$ inside its open complement. Thus, it is necessary to find the minimum $k_{\min }^{-}$and the maximum $k_{\max }^{+}$such that $\mathscr{P}\left(e^{i \theta}, k\right) \neq$ 0 , for all $0<\theta<2 \pi$ and for all $k \in\left(k_{\min }^{-}, k_{\max }^{+}\right)$and keep the quantity of roots in each zone. If $k$ satisfies $\mathscr{P}\left(e^{i \theta}, k\right)=0$, then $k \notin\left(k_{\min }^{-}, k_{\max }^{+}\right)$and therefore we seek the maximum $k<0$ and the minimum $k>0$ such that $\mathscr{P}\left(e^{i \theta}, k\right)=0$; that is,

$$
G(\theta)+k F(\theta)+i k H(\theta)=0 .
$$

for some $0 \leq \theta \leq 2 \pi$ and $k \neq 0$. Consequently, the following system must be solved:

$$
\begin{aligned}
G(\theta)+k F(\theta) & =0 \\
k H(\theta) & =0 .
\end{aligned}
$$

For each $\theta_{l} \in \sigma(H)$, the before system has solution by taking $k=-G\left(\theta_{l}\right) / F\left(\theta_{l}\right)$. Now, if $z \in \partial \mathscr{D}$, then $z^{-1}=\bar{z}$, and then $G(\theta)=\left|p_{A}\left(e^{i \theta}\right)\right|^{2}>0$. Consequently, $-G(\theta) / F(\theta)$ is positive if $F(\theta)<0$ and $-G(\theta) / F(\theta)$ is negative if $F(\theta)>0$. Notice that all the values of $k$ satisfying system (19) are determined by the zeroes of $H(\theta)$. Therefore, $k=-G\left(\theta_{l}\right) / F\left(\theta_{l}\right)$ is positive if $F\left(\theta_{l}\right) \in K^{-}$and it is negative if $F\left(\theta_{l}\right) \in K^{+}, \theta_{l} \in \sigma(H)$. Thence, the maximum $k<0$ and minimum $k>0$ satisfying $G\left(\theta_{l}\right)+k F\left(\theta_{l}\right)+i k H\left(\theta_{l}\right)=0$ for $\theta_{l} \in \sigma(H)$ are, respectively, given by

$$
\begin{aligned}
& k_{\min }^{-}=\max \left\{-\frac{G\left(\theta_{l}\right)}{F\left(\theta_{l}\right)} \mid F\left(\theta_{l}\right) \in K^{+}\right\}, \\
& k_{\max }^{+}=\min \left\{-\frac{G\left(\theta_{l}\right)}{F\left(\theta_{l}\right)} \mid F\left(\theta_{l}\right) \in K^{-}\right\} .
\end{aligned}
$$

Finally, if $K^{-}=\left\{0^{-}\right\}\left(K^{+}=\left\{0^{+}\right\}\right.$, resp. $)$, then $k_{\max }^{+}=$ $\lim _{F \rightarrow 0^{-}}(-G(\theta) / F)=+\infty\left(k_{\text {min }}^{-}=\lim _{F \rightarrow 0^{+}}(-G(\theta) / F)=-\infty\right.$, resp.), and this ends the proof.

Remark 9. If we suppose that $G(\theta)+k F(\theta)+i k H(\theta)=0$, zeroes $\theta_{l} \in \sigma(H)$ must not be considered such that $F\left(\theta_{l}\right)=$ 0 ; if so, then $0=G\left(\theta_{l}\right)+k F\left(\theta_{l}\right)+i k H\left(\theta_{l}\right)=G\left(\theta_{l}\right)$, no matter the values of $k$, which is a contradiction since $G(\theta)=$ $\left|p_{A}\left(e^{i \theta}\right)\right|^{2} \neq 0$ for all $\theta \in[0,2 \pi]$, by the fact that $p_{A}(z)$ is a Schur polynomial.

Remark 10. Note that $\left(k_{\min }^{-}, k_{\max }^{+}\right)$extends any interval $(a, b)$ for the uncertainty $k$ to keep stability of the ray $P(z, k)$ and such that the family $\mathscr{P}(z, k)$ satisfies the zero exclusion principle in the closure $[a, b]$.

Example 11. Consider the discrete-time system

$$
x_{m+1}=\left[\begin{array}{ccc}
0 & 1 & 0 \\
0 & 0 & 1 \\
0 & \frac{1}{4} & 0
\end{array}\right] x_{m}+\left[\begin{array}{l}
0 \\
0 \\
1
\end{array}\right] u_{m},
$$

having as characteristic polynomial to $p_{A}(z)=z^{3}-(1 / 4) z$, which is trivially Schur stable. If we need to design the feedback $u_{m}=-k(1,2,1) x_{m}$, then we obtain the polynomial $p_{c}(z)=z^{2}+2 z+1$ and the polynomial family $P(z, k)=$ $p_{A}(z)+k p_{c}(z)$. We will now compute the maximal stability interval with our proposed method. Evaluate the polynomials $p_{A}(z)$ and $p_{c}(z)$ in the unit circle to obtain

$$
\begin{aligned}
p_{A}\left(e^{i \theta}\right) & =e^{3 i \theta}-\frac{1}{4} e^{i \theta} \\
& =\cos 3 \theta-\frac{1}{4} \cos \theta+i\left(\sin 3 \theta-\frac{1}{4} \sin \theta\right) \\
& =R(\theta)+i Q(\theta), \\
p_{c}\left(e^{i \theta}\right) & =e^{2 i \theta}+2 e^{i \theta}+1 \\
& =\cos 2 \theta+2 \cos \theta+1+i(\sin 2 \theta+2 \sin \theta) \\
& =r(\theta)+i q(\theta)
\end{aligned}
$$

Thus,

$$
\begin{aligned}
& F(\theta)=\cos 3 \theta+2 \cos 2 \theta+\frac{1}{2} \cos \theta-\frac{1}{2} \\
& G(\theta)=\frac{17}{16}-\frac{1}{2} \cos 2 \theta \\
& H(\theta)=-\sin 3 \theta-2 \sin 2 \theta-\sin \theta
\end{aligned}
$$

Note that just zeroes of $H \bmod (2 \pi)$ must be considered. Now, it is not hard to see that $H(\theta)$ can be written as

$$
H(\theta)=-4 \sin \theta \cos \theta(\cos \theta+1),
$$

and, thence, its zeroes set is $\sigma(H)=\{0, \pi / 2, \pi, 3 \pi / 2\}$. One can see that $F(\pi)=0$; therefore $\theta=\pi$ will not be considered in $K^{-}$and $K^{+}$. Next,

$$
\begin{aligned}
F(0) & =3 ; \\
F\left(\frac{\pi}{2}\right) & =-2.5 ; \\
F\left(\frac{3 \pi}{2}\right) & =-2.5 ; \\
G(0) & =0.5625 ; \\
G\left(\frac{\pi}{2}\right) & =1.5625 ; \\
G\left(\frac{3 \pi}{2}\right) & =1.5625 .
\end{aligned}
$$

Therefore,

$$
\begin{aligned}
& k_{\min }^{-}=-\frac{G(0)}{F(0)}=-0.1875 \\
& k_{\max }^{+}=-\frac{G(\pi / 2)}{F(\pi / 2)}=0.625 .
\end{aligned}
$$

Finally, system (19) is stable for all $k \in(-0.1875,0.625)$. That is, all of its poles lie inside the unit circle for all $k \in$ $(-0.1875,0.625)$. 

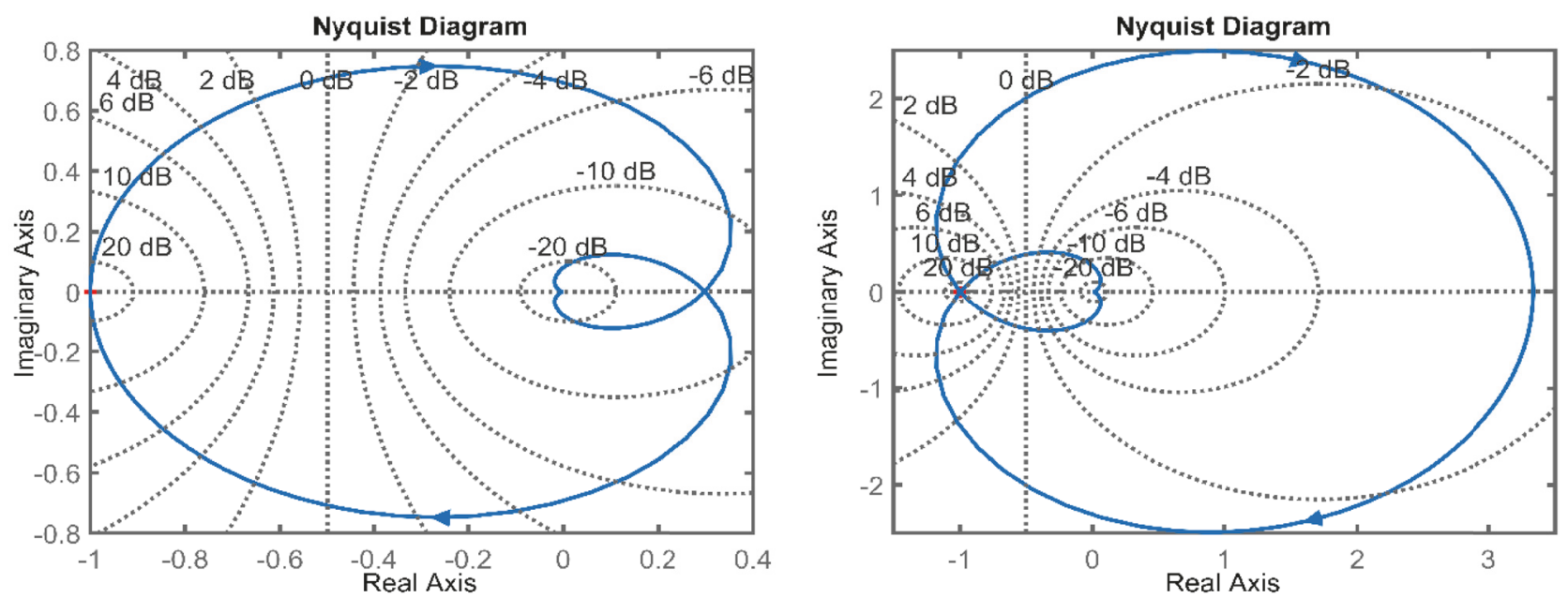

FIGURE 1: Nyquist diagram of the closed-loop system for $k_{\min }^{-}$(left image) and $k_{\max }^{+}$(right image) extremes of the maximal stability interval.
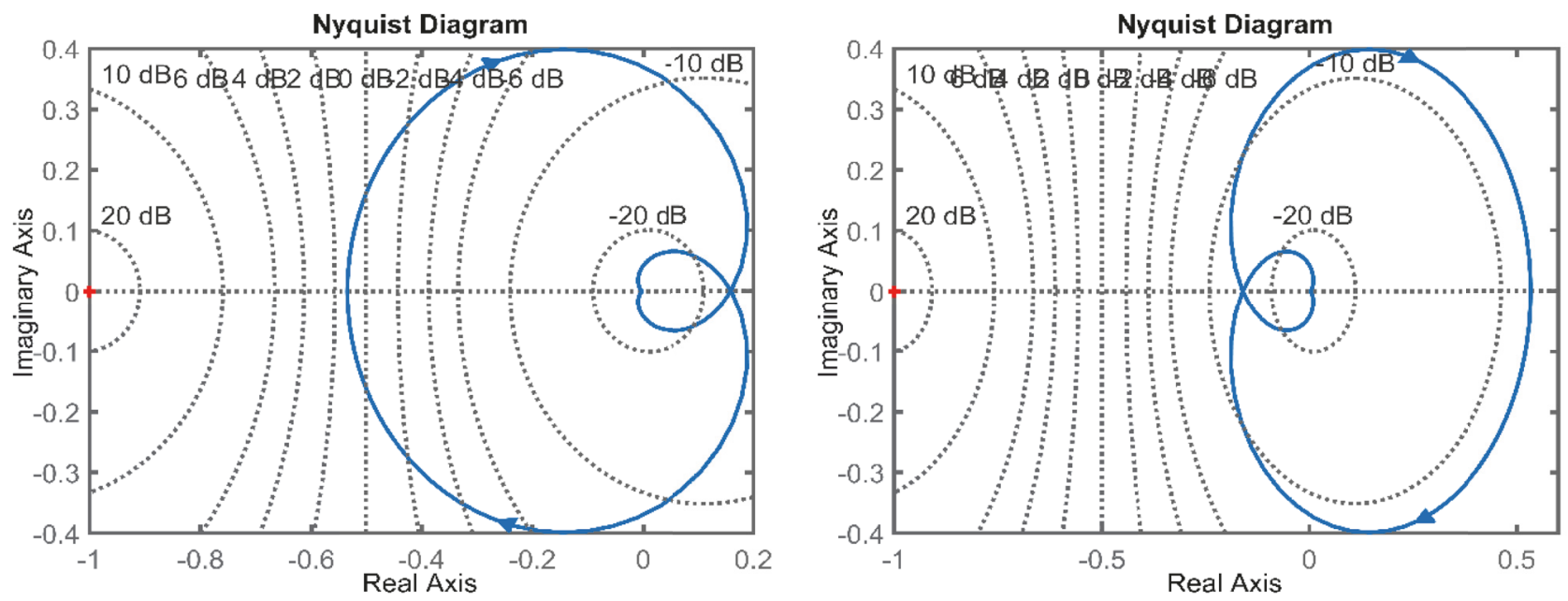

Figure 2: Nyquist diagram for $k=-0.1$ (left image) and $k=0.1$ (right image).

Recall that the characteristic equation $p_{A}(z)$ is Schur stable. In Figure 1, one can see the Nyquist diagram intersecting the point of $-1+0 \mathrm{i}$ for the extreme values of the maximal stability interval obtaining instability in the system, while, in Figure 2, stability for the system arises for values of the gain within the interval of robustness; moreover, in Figure 3 the point $-1+0 \mathrm{i}$ is encircled by the Nyquist diagram for a value not in the computed interval. Finally, robust stability is depicted by the set values in Figure 4, since origin is excluded and there is a Schur stable polynomial member of the family $P(z, k)$ for $k=0$.

5.3. The Roots Criterion for Discrete Systems with Single TimeDelay Control. Consider the discrete-time system with a single time-delay

$$
x_{m+1}=A x_{m}+B x_{m-\tau}, \quad \tau \in \mathbb{N},
$$

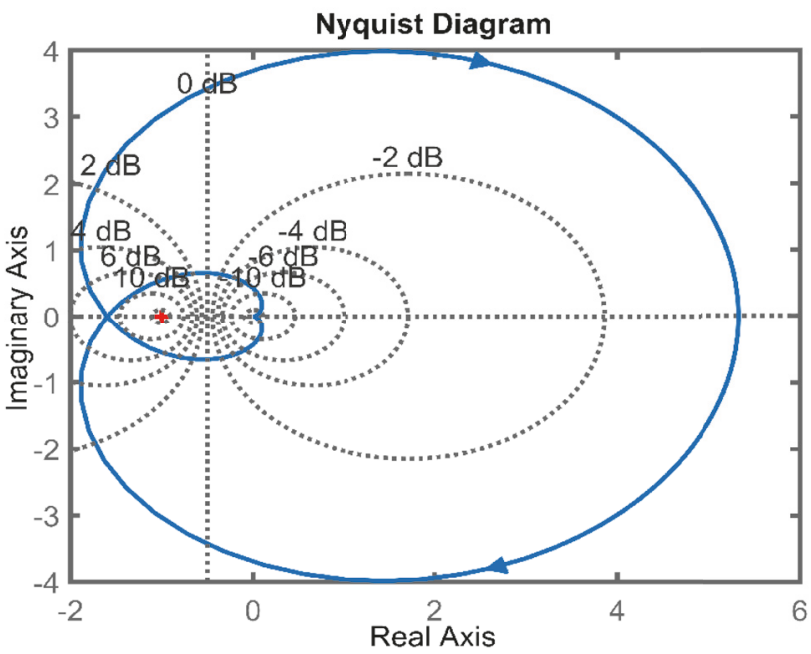

FIGURE 3: Nyquist diagram for $k=1$. 

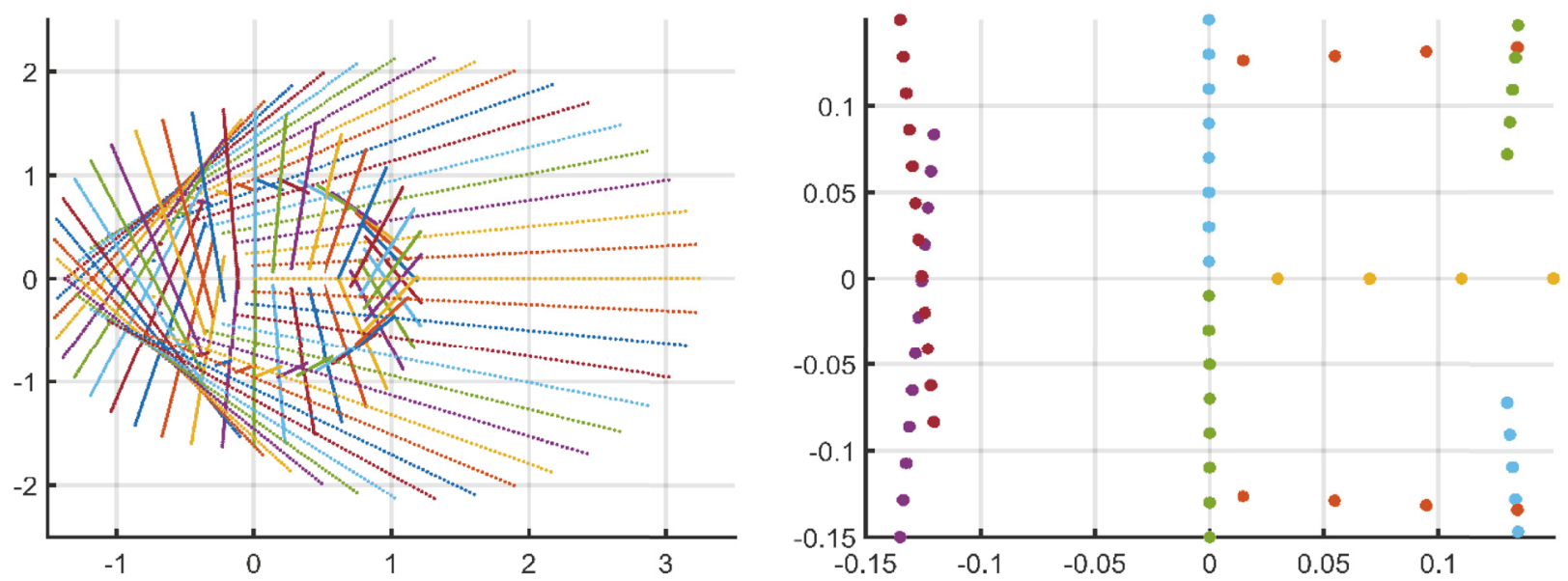

FIGURE 4: Set value of the family $P(z, k)=z^{3}+k z^{2}+2 k z+(k-1 / 4)$ with $k \in(-0.1875,0.625)$; right image with zoom around the origin.

with $A, B \in \mathbb{R}^{n \times n}$, whose stability is in charge of the roots of its characteristic equation given by $p(z)=\operatorname{det}\left(z^{\tau+1} I-z^{\tau} A-B\right)=$ 0 [24].

Based on the aforementioned reference, we shall consider the control system

$$
x_{m+1}=A x_{m}+b u_{m-\tau}, \quad \tau \in \mathbb{N},
$$

where

$$
\begin{aligned}
& A=\left[\begin{array}{ccccc}
0 & 1 & 0 & \cdots & 0 \\
0 & 0 & 1 & \cdots & 0 \\
\vdots & \vdots & \vdots & \ddots & \vdots \\
0 & 0 & 0 & \cdots & 1 \\
-a_{n} & -a_{n-1} & -a_{n-2} & \cdots & -a_{1}
\end{array}\right], \\
& b=\left[\begin{array}{c}
0 \\
0 \\
\vdots \\
1
\end{array}\right]
\end{aligned}
$$

for which the linear delayed control $u_{m-\tau}=-k c^{T} x_{m-\tau}$, with $c^{T}=\left(c_{n}, \ldots, c_{1}\right)$, endows the system (27) with a single timedelay as required in system (26).

Lemma 12. The closed-loop characteristic equation of system (27) is given by the ray $P_{\tau}(z, k)=z^{n \tau} p_{A}(z)+k z^{n} p_{c}(z)$, where $p_{A}(z)=\operatorname{det}(z I-A)$ and $p_{c}(z)=c_{1} z^{n-1}+\cdots+c_{n-1} z+c_{n}$.

Proof. If we closed-loop system (27) with the control $u_{m-\tau}=$ $-k c^{T} x_{m-\tau}$ the following arises:

$$
x_{m+1}=A x_{m}-k b c^{T} x_{m-\tau},
$$

and by taking $B(k)=-k b c^{T}$, we get that the characteristic equation is

$$
\begin{aligned}
\operatorname{det} & \left(z^{\tau+1} I-z^{\tau} A-B(k)\right) \\
= & \operatorname{det}\left(z^{\tau}\left[z I-A-z^{-\tau} B(k)\right]\right) \\
= & \operatorname{det}\left(z^{\tau}\left[z I-A+k z^{-\tau} b c^{T}\right]\right) \\
= & z^{n \tau} \operatorname{det}\left(z I-\left(A-k z^{-\tau} b c^{T}\right)\right) \\
= & z^{n \tau}\left[p_{A}(z)+k z^{-\tau} p_{c}(z)\right] \\
= & z^{n \tau} p_{A}(z)+k z^{n} p_{c}(z),
\end{aligned}
$$

as we claim.

In the same essence as the technique in single time case, by evaluating in the unit complex circle we get

$$
\begin{gathered}
\tilde{p}_{A}\left(e^{i \theta}\right)=e^{i n \tau \theta} p_{A}\left(e^{i \theta}\right)=e^{i n \tau \theta}[R+i Q](\theta) \\
\tilde{p}_{c}\left(e^{i \theta}\right)=e^{i n \theta} p_{c}\left(e^{i \theta}\right)=e^{i n \theta}[r+i q](\theta)
\end{gathered}
$$

where $R, Q, r$, and $q$ are the functions given in (7). The product to consider in this case is $\mathscr{P}_{\tau}(z, k)=\widetilde{p}_{A}\left(z^{-1}\right) P_{\tau}(z, k)$. Thus, $\mathscr{P}_{\tau}(z, k)$ in $z=e^{i \theta}$ can be written as

$$
\begin{aligned}
& \mathscr{P}_{\tau}\left(e^{i \theta}, k\right)=\widetilde{p}_{A}\left(e^{-i \theta}\right) \tilde{p}_{A}\left(e^{i \theta}\right)+\widetilde{p}_{A}\left(e^{-i \theta}\right) \tilde{p}_{c}\left(e^{i \theta}\right) \\
& \quad=e^{-i n \tau \theta} p_{A}\left(e^{-i \theta}\right) e^{i n \tau \theta} p_{A}\left(e^{i \theta}\right)+e^{-i n \tau \theta} p_{A}\left(e^{-i \theta}\right) \\
& \quad \cdot e^{i n \theta} p_{c}\left(e^{i \theta}\right)=\left[R^{2}+Q^{2}\right](\theta) \\
& +k[\cos (\tau \theta)[R r+Q q](\theta) \\
& +\sin (\tau \theta)[R q-Q r](\theta)] \\
& +i k[\cos (\tau \theta)[R q-Q r](\theta) \\
& -\sin (\tau \theta)[R r+Q q](\theta)] .
\end{aligned}
$$


Define

$$
\begin{aligned}
F_{\tau}(\theta)= & \cos (\tau \theta)[R r+Q q](\theta) \\
& +\sin (\tau \theta)[R q-Q r](\theta) \\
= & \cos (\tau \theta) F(\theta)+\sin (\tau \theta) H(\theta), \\
H_{\tau}(\theta)= & \cos (\tau \theta)[R q-Q r](\theta) \\
& -\sin (\tau \theta)[R r+Q q](\theta) \\
= & \cos (\tau \theta) H(\theta)-\sin (\tau \theta) F(\theta), \\
G_{\tau}(\theta)= & G(\theta)
\end{aligned}
$$

$F, G$, and $H$ are the functions defined in (10). Note that if $\tau=$ 0 , we recover the single time case from the previous section. Write then the family $\mathscr{P}_{\tau}\left(e^{i \theta}, k\right)$ in the form

$$
\mathscr{P}_{\tau}\left(e^{i \theta}, k\right)=G_{\tau}(\theta)+k F_{\tau}(\theta)+i k H_{\tau}(\theta),
$$

for $G(\theta)$ defined in (10). Analogous to the sets $K^{-}$and $K^{+}$ from (14), let us define the sets

$$
\begin{aligned}
& \left.K_{\tau}^{+}=\left\{F_{\tau}\left(\theta_{l}\right) \mid \theta_{l} \in \sigma\left(H_{\tau}\right), F_{\tau}\left(\theta_{l}\right)>0\right)\right\} \\
& \left.K_{\tau}^{-}=\left\{F_{\tau}\left(\theta_{l}\right) \mid \theta_{l} \in \sigma\left(H_{\tau}\right), F_{\tau}\left(\theta_{l}\right)<0\right)\right\} .
\end{aligned}
$$

Thence, the following result is held.

Theorem 13. Let $p_{A}(z)=z^{n}+a_{1} z^{n-1}+\cdots+a_{n}$ be a real Schur polynomial and consider any real polynomial $p_{c}(z)=$ $c_{1} z^{m}+\cdots+c_{m} z+c_{m+1}$, with $n>m$. Then, the maximal Schur stability interval for the monoparametric polynomial $P_{\tau}(z, k)=$ $z^{n \tau} p_{A}(z)+k z^{n} p_{c}(z)$ is $\left(k_{\min }^{-}, k_{\max }^{+}\right)$, where

$$
\begin{aligned}
& k_{\min }^{-}=\max \left\{-\frac{G_{\tau}\left(\theta_{l}\right)}{F_{\tau}\left(\theta_{l}\right)} \mid F_{\tau}\left(\theta_{l}\right) \in K_{\tau}^{+}\right\} \\
& k_{\max }^{+}=\min \left\{-\frac{G_{\tau}\left(\theta_{l}\right)}{F_{\tau}\left(\theta_{l}\right)} \mid F_{\tau}\left(\theta_{l}\right) \in K_{\tau}^{-}\right\} .
\end{aligned}
$$

Proof. It is analogous to the proof of Theorem 8.

Remark 14. As in Remark 9, we must not consider zeroes $\theta_{l} \in$ $\sigma\left(H_{\tau}\right)$ such that $F_{\tau}\left(\theta_{l}\right)=0$, since

$$
G_{\tau}\left(\theta_{l}\right)+k F_{\tau}\left(\theta_{l}\right)+i k H_{\tau}\left(\theta_{l}\right)=G_{\tau}\left(\theta_{l}\right)=G\left(\theta_{l}\right)=0,
$$

which is a contradiction due to the fact that $G(\theta)=$ $\left|p_{A}\left(e^{i \theta}\right)\right|^{2} \neq 0$ for all $\theta \in[0,2 \pi]$, by the fact that $p_{A}(z)$ is a Schur polynomial.

Example 15 (Phillips and Nagle (1995) [4]). Consider the digital system described by the difference equation

$$
y[m+2]-1.7 y[m+1]+0.72 y[m]=u[m-\tau] .
$$

Then, the change of variables $x_{1}(m)=y[m]$ and $x_{2}(m)=$ $x_{1}(m+1)=y[m+1]$ transforms the difference equation (38) in to the states variable system

$$
\begin{aligned}
& x_{1}(m+1)=x_{2}(m) \\
& x_{2}(m+1)=-0.72 x_{1}(m)+1.7 x_{2}(m)+u_{m-\tau}
\end{aligned}
$$

We may express these equations in state variable representation as

$$
x_{m+1}=\left[\begin{array}{cc}
0 & 1 \\
-0.72 & 1.7
\end{array}\right] x_{m}+\left[\begin{array}{l}
0 \\
1
\end{array}\right] u_{m-\tau},
$$

where $x_{m}^{T}=\left(x_{1}(m), x_{2}(m)\right)$ and $u_{m-\tau}=u[m-\tau]$. The openloop characteristic polynomial of the system is $p_{A}(z)=z^{2}-$ $1.7 z+0.72$ with roots $z_{1}=0.9$ and $z_{2}=0.8$, which indicates that the open-loop system is Schur stable. Let us design the feedback

$$
u_{m-\tau}(x, k)=-k\left[1.5 x_{1}(m-\tau)+0.8 x_{2}(m-\tau)\right]
$$

to obtain the polynomial $p_{c}(z)=0.8 z+1.5$ and the closedloop characteristic polynomial family

$$
\begin{aligned}
P_{\tau}(z, k) & =z^{2 \tau} p_{A}(z)+k z^{2} p_{c}(z) \\
& =z^{2 \tau}\left(z^{2}-1.7 z+0.72\right)+k z^{2}(0.8 z+1.5)
\end{aligned}
$$

Thence, by evaluating $z=e^{i \theta}, \theta \in[0,2 \pi]$, the following complex functions arise:

$$
\begin{aligned}
p_{A}\left(e^{i \theta}\right)= & e^{i 2 \theta}-1.7 e^{i \theta}+0.72 \\
= & \cos 2 \theta-1.7 \cos \theta+0.72 \\
& +i[\sin 2 \theta-1.7 \sin \theta]=R(\theta)+i Q(\theta) ; \\
p_{c}\left(e^{i \theta}\right)= & 0.8 e^{i \theta}+1.5=0.8 \cos \theta+1.5+i 0.8 \sin \theta \\
= & r(\theta)+i q(\theta),
\end{aligned}
$$

and, consequently,

$$
\begin{aligned}
F(\theta) & =[R r+Q q](\theta) \\
& =3 \cos ^{2} \theta-1.174 \cos \theta-1.78 \\
H(\theta) & =[R q-Q r](\theta)=\sin \theta[-3 \cos \theta+2.326] \\
G(\theta) & =\left[R^{2}+Q^{2}\right](\theta) \\
& =2.88 \cos ^{2} \theta-5.848 \cos \theta+2.9684
\end{aligned}
$$

Therefore, if $\tau=2$ the directly involved functions $F_{\tau}, G_{\tau}$, and $H_{\tau}$ are given by

$$
\begin{aligned}
& F_{\tau}(\theta)=\cos (\tau \theta) F(\theta)+\sin (\tau \theta) H(\theta) \\
& =9 \cos ^{4} \theta+168.17 \cos ^{3} \theta-12.56 \cos ^{2} \theta \\
& \quad+5.826 \cos \theta+1.78 \\
& H_{\tau}(\theta)=\cos (\tau \theta) H(\theta)-\sin (\tau \theta) F(\theta) \\
& \quad=\sin \theta\left[-12 \cos ^{3} \theta+7 \cos ^{2} \theta+6.56 \cos \theta-2.326\right] \\
& G_{\tau}(\theta)=G(\theta) .
\end{aligned}
$$

The change of variable $x=\cos \theta$ in the function $-12 \cos ^{3} \theta+$ $7 \cos ^{2} \theta+6.56 \cos \theta-2.326=0$ arises at the roots $x_{1}=$ 

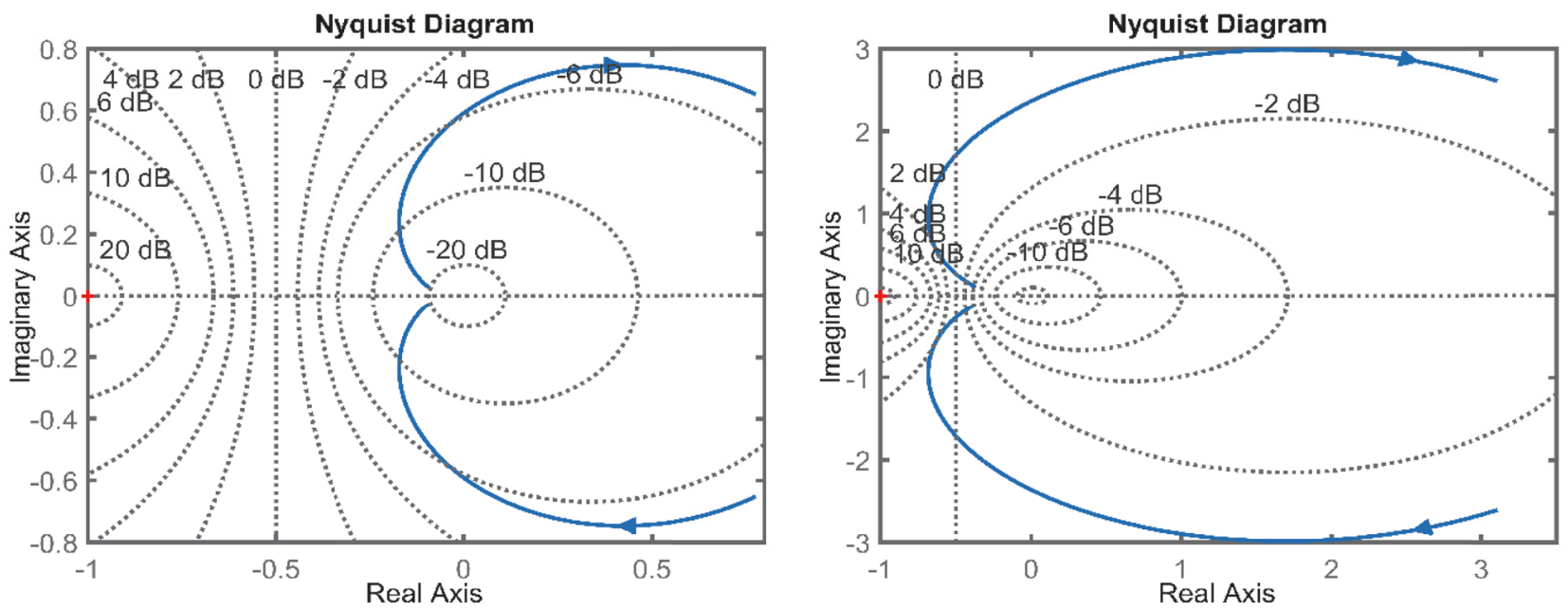

FIGURE 5: Nyquist diagram for $k=0.01$ (left image) and $k=0.04$ (right image).

0.94479, $x_{2}=0.30693$, and $x_{3}=-0.6684$. Consequently, $\theta_{1}=\cos ^{-1}\left(x_{1}\right), \theta_{2}=\cos ^{-1}\left(x_{2}\right)$, and $\theta_{3}=\cos ^{-1}\left(x_{3}\right)$ are real zeroes of $H_{\tau}$. Moreover, it is not hard to see that $\theta_{3}=0$ and $\theta_{4}=\pi$ are also roots of $H_{\tau}$. Thence,

$$
\begin{gathered}
F_{\tau}(0)=172.046, \\
G_{\tau}(0)=0.0004 ; \\
F_{\tau}(\pi)=-175.776, \\
G_{\tau}(\pi)=11.6964 ; \\
F_{\tau}\left(\theta_{1}\right)=145.07198, \\
G_{\tau}\left(\theta_{1}\right)=3.01403 ; \\
F_{\tau}\left(\theta_{2}\right)=7.32785, \\
G_{\tau}\left(\theta_{2}\right)=4.44474 ; \\
F_{\tau}\left(\theta_{3}\right)=-56.14728, \\
G_{\tau}\left(\theta_{3}\right)=11.16388 .
\end{gathered}
$$

Therefore,

$$
\begin{aligned}
k_{\min }^{-} & =\max \left\{-\frac{G(0)}{F(0)},-\frac{G\left(\theta_{1}\right)}{F\left(\theta_{1}\right)},-\frac{G\left(\theta_{2}\right)}{F\left(\theta_{2}\right)}\right\} \\
& =\max \{-0.00000232,-0.02077,-0.60655\} \\
& =-0.00000232 . \\
k_{\max }^{+} & =\min \left\{-\frac{G(\pi)}{F(\pi)},-\frac{G\left(\theta_{3}\right)}{F\left(\theta_{3}\right)}\right\} \\
& =\min \{0.06654,0.19883\}=0.06654 .
\end{aligned}
$$

Consequently, the discrete system (38), with the delayed time feedback

$$
u_{m-2}(x, k)=-k\left[1.5 x_{1}(m-2)+0.8 x_{2}(m-2)\right],
$$

has all of its roots inside the unit complex disk (and then, stable)

$$
k \in K=(-0.00000232,0.06654) \text {. }
$$

Since the extremes of the maximal stability interval $K$ are so close to each other, a Nyquist diagram becomes sensible under the choice of gains. Consequently, it is hard to see that the Nyquist plot passes throught the point $-1+0 \mathrm{i}$. However, since the closed-loop characteristic equation $z^{4} p_{A}(z)$ is Schur stable, in Figure 5 stability is depicted by Nyquist diagram ($1+0 \mathrm{i}$ point not enclosed) where values of the gain $k$ belong to $K$ and in Figure 6 instability (-1+0i point enclosed) is depicted for gains not in $K$. In Figure 7, one can see that the origin is excluded from the set values for $k \in(-0.00000232,0.06654)$ and since $P_{\tau=2}(z, 0)$ is Schur stable, then the whole polynomial family $P_{\tau=2}(z, k)$ is robustly stable.

\section{Conclusions}

In this work, a linear feedback control has been designed to obtain robust stability in discrete-time control systems and although the stability regions of both analogue and discrete systems are different, we were able to adapt a technique which is given for analogue systems to find the maximal Schur stability interval for a ray of polynomials emerging from the closed-loop discrete control system. A pair of examples have been developed to illustrate the functionality of the technique. Finally, the obtained technique has been analogously used for discrete control systems with a single time-delay controller, which comes to be an easy algebraic stability test.

\section{Conflicts of Interest}

The authors declare that there are no conflicts of interest of any kind regarding the publication of this paper. 

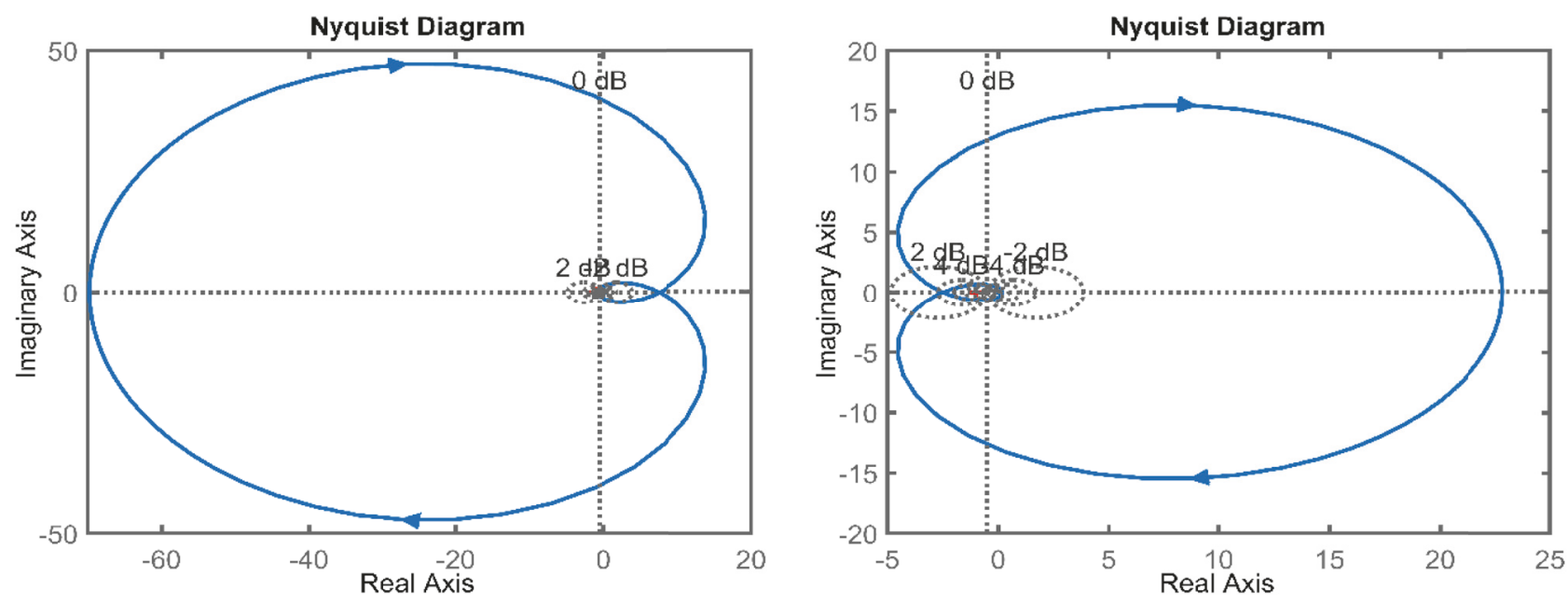

FIGURE 6: Nyquist diagram for $k=-0.60655$ (left image) and $k=0.19883$ (right image).
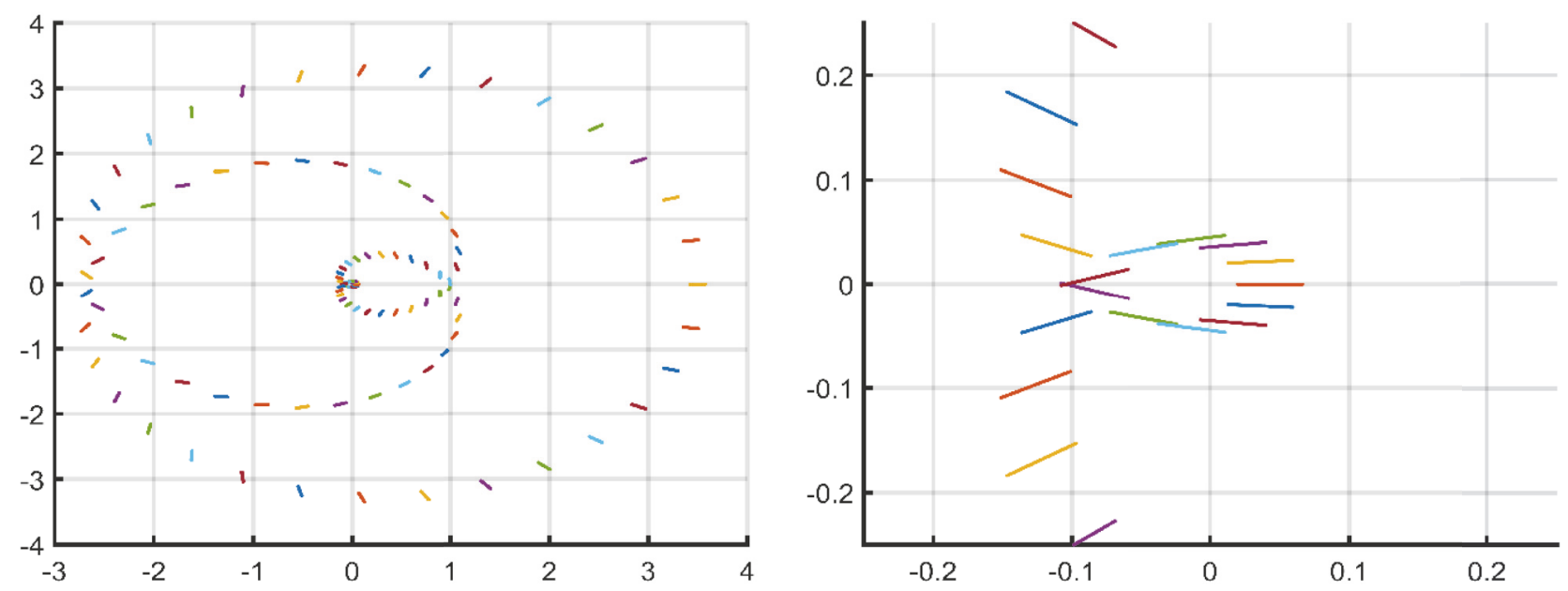

Figure 7: Set value of the polynomial family $P_{\tau=2}(z, k)=z^{4}\left(z^{2}-1.7 z+0.72\right)+k z^{2}(0.8 z+1.5)$ with $k \in(-0.00000232,0.06654)$; right image with zoom around the origin.

\section{Acknowledgments}

The first author is supported by CONACYT as a Ph.D. fellowship and second author as CONACYT chair professor. López-Rentería wants to acknowledge CONACYT, Grant no. 290941-UIA, as well as thank Heba Mohamed, MPE Editor, for the invited contribution call.

\section{References}

[1] P. A. Moya, Señales y Sistemas: Fundamentos Matemáticos, CDMB, Inst. Tec. de Costa Rica, Costa Rica, 1st edition, 2008.

[2] K. Ogata, Discrete-Time Control Systems, Prentice Hall, Englewood Cliffs, New Jersey, NJ, USA, 2nd edition, 1995.

[3] A. V. Oppenheim, A. S. Willsky, and I. T. Young, Signals and Systems, Signal Processing Series, Prentice Hall, 1983.

[4] C. L. Phillips and H. T. Nagle, Digital Control System Analysis and Design, vol. 3rd, Prentice Hall, Englewood Cliffs, New Jersey, NJ, USA, 1995.
[5] B. R. Barmish, New Tools for Robustness of Linear Systems, Macmillan, New York, NY, USA, 1994.

[6] S. P. Bhattacharyya, H. Chapellat, and L. H. Keel, Robust Control: The parametric Approach, Prentice Hall, Upper Saddle River, New Jersey, NJ, USA, 1995.

[7] D. Hinrichsen and A. J. Pritchard, "Mathematical Systems Theory I," in Modeling, State Space Analysis, Stability and Robustness, vol. 48 of Texts in Applied Mathematics, Springer, Berlin, Germany, 2005.

[8] J.-A. López-Renteria, B. Aguirre-Hernández, and F. Verduzco, "The boundary crossing theorem and the maximal stability interval," Mathematical Problems in Engineering, vol. 2011, Article ID 123403, 13 pages, 2011.

[9] E. I. Jury, "A Simplified Stability Criterion for Linear Discrete Systems," Proceedings of the IRE, vol. 50, no. 6, pp. 1493-1500, 1962.

[10] E. I. Jury, "A Note on the Modified Stability Table for Linear Discrete Time Systems," IEEE Transactions on Circuits and Systems II: Express Briefs, vol. 38, no. 2, pp. 221-223, 1991. 
[11] J. Schur, "On series which are bounded in the unit circle," Journal für die reine und angewandte Mathematik, vol. 147, no. 4, pp. 205-232, 1917.

[12] A. Cohn, "Uber die Anzahl der Wurzeln einer algebraischen Gleichung in einem Kreise," Mathematische Zeitschrift, vol. 2, pp. 110-148, 1922.

[13] M. Benidir, "Modified Schur-Cohns algorithm and Jurys test in the complex case," in In Fundamentals of Discrete-Time Systems, pp. 195-202, TSI Press, 1993.

[14] M. Benidir, "On the root distribution of general polynomials with respect to the unit circle," Signal Processing, vol. 53, no. 1, pp. 75-82, 1996.

[15] R. Greiner, "Necessary conditions for Schur-stability of interval polynomials," Institute of Electrical and Electronics Engineers Transactions on Automatic Control, vol. 49, no. 5, pp. 740-744, 2004.

[16] A. C. Bartlett, C. V. Hollot, and H. Lin, "Root locations of an entire polytope of polynomials: it suffices to check the edges," Mathematics of Control, Signals, and Systems, vol. 1, no. 1, pp. 61-71, 1988.

[17] J. E. Ackermann and B. R. Barmish, "Robust Schur stability of a polytope of polynomials," Institute of Electrical and Electronics Engineers Transactions on Automatic Control, vol. 33, no. 10, pp. 984-986, 1988.

[18] R. Matusu, R. Prokop, and J. Korbel, "Description and analysis of interval systems," in Proceedings of the 23rd European Conference on Modelling and Simulation, ECMS '09, vol. 23, pp. 551556, June 2009.

[19] R. Matusu, "Robust stability analysis of discrete-time systems with parametric uncertainty: a graphical approach," International Journal of Mathematical Models and Methods in Applied Sciences, vol. 8, pp. 95-102, 8.

[20] B. Aguirre-Hernández, R. García-Sosa, H. Leyva-Castellanos et al., "Conditions for the Schur stability of segments of polynomials of the same degree," Boletín de la Sociedad Matemática Mexicana, vol. 21, no. 2, pp. 309-321, 2015.

[21] S. Bialas, "A necessary and sufficient condition for the stability of convex combinations of stable polynomials or matrices," Bulletin of the Polish Academy of Sciences-Technical Sciences, vol. 33, no. 9-10, pp. 473-480, 1985.

[22] B. Aguirre-Hernández, E. Campos-Cantón, J. A. LópezRenteria, and E. C. Díaz González, "A polynomial approach for generating a monoparametric family of chaotic attractors via switched linear systems," Chaos, Solitons \& Fractals, vol. 71, pp. 100-106, 2015.

[23] F. R. García, B. Aguirre, and R. Suárez, "Stabilization of linear sampled-data systems by a time-delay feedback control," Mathematical Problems in Engineering, vol. 2008, Article ID 270518, 15 pages, 2008.

[24] J. Diblík, D. Y. Khusainov, J. Baštinec, and A. S. Sirenko, "Exponential stability of linear discrete systems with constant coefficients and single delay," Applied Mathematics Letters, vol. 51, pp. 68-73, 2016. 


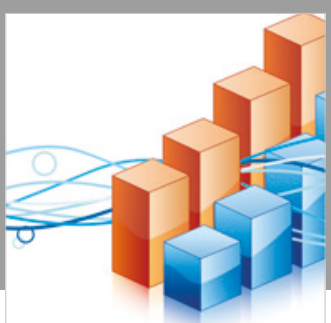

Advances in

Operations Research

\section{-n-m}
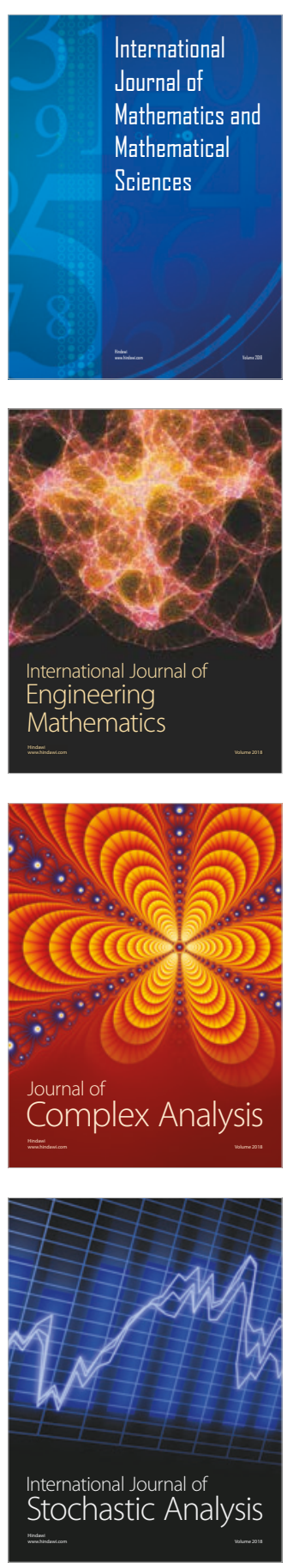
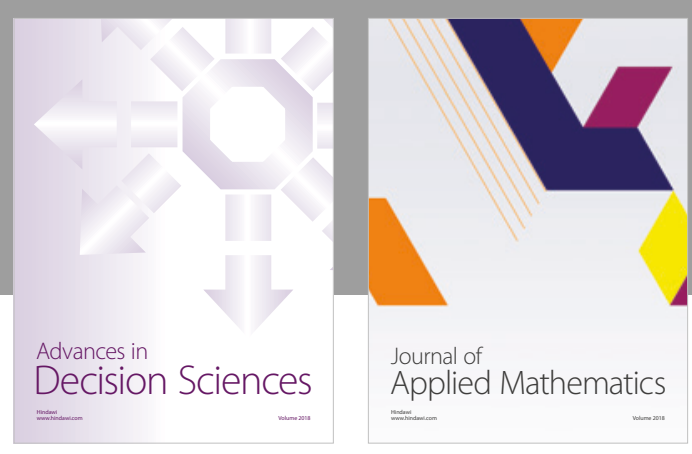

Journal of

Applied Mathematics
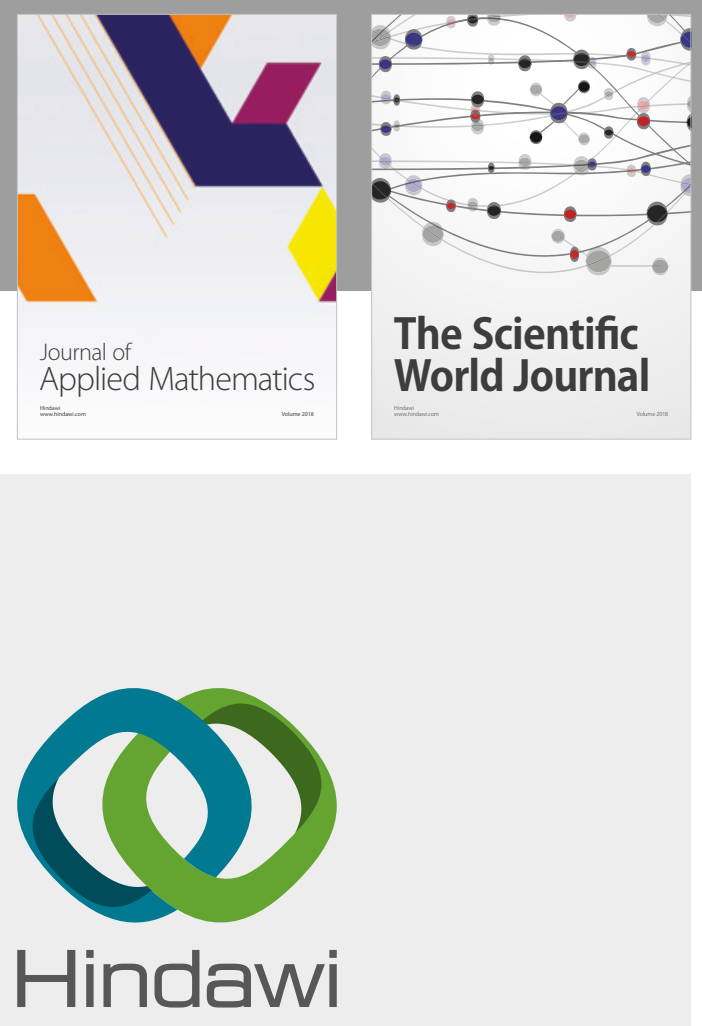

Submit your manuscripts at

www.hindawi.com

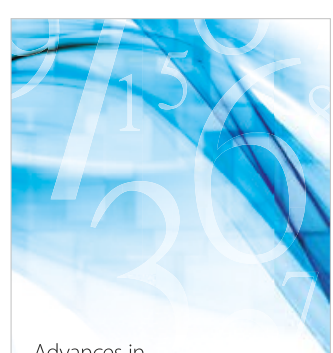

Advances in
Numerical Analysis
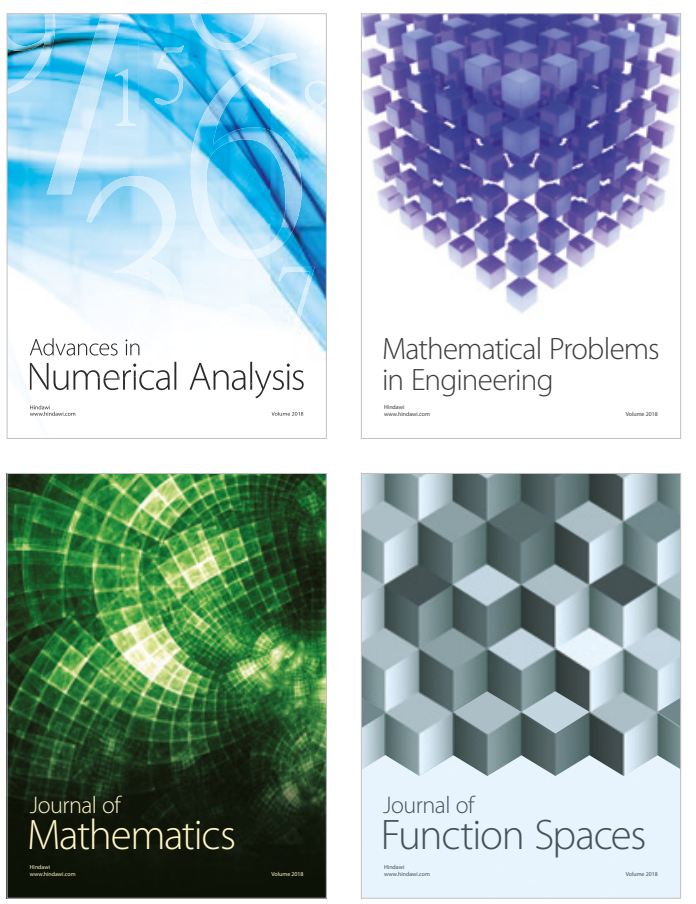

Mathematical Problems in Engineering

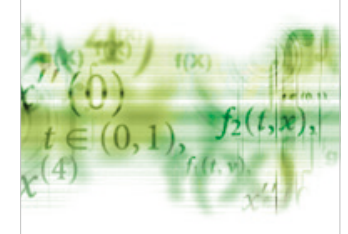

International Journal of

Differential Equations

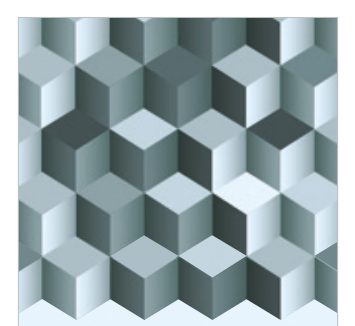

Journal of

Function Spaces

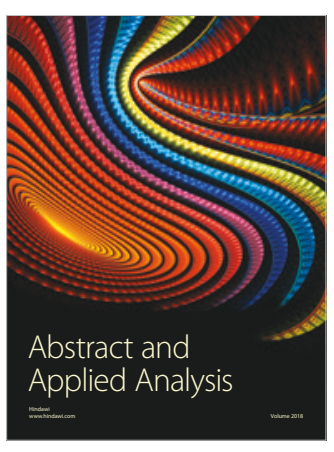

The Scientific

World Journal

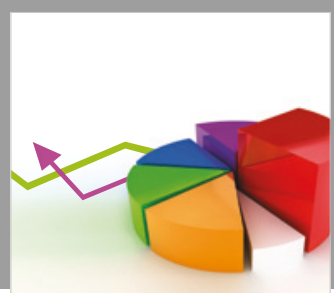

Journal of

Probability and Statistics
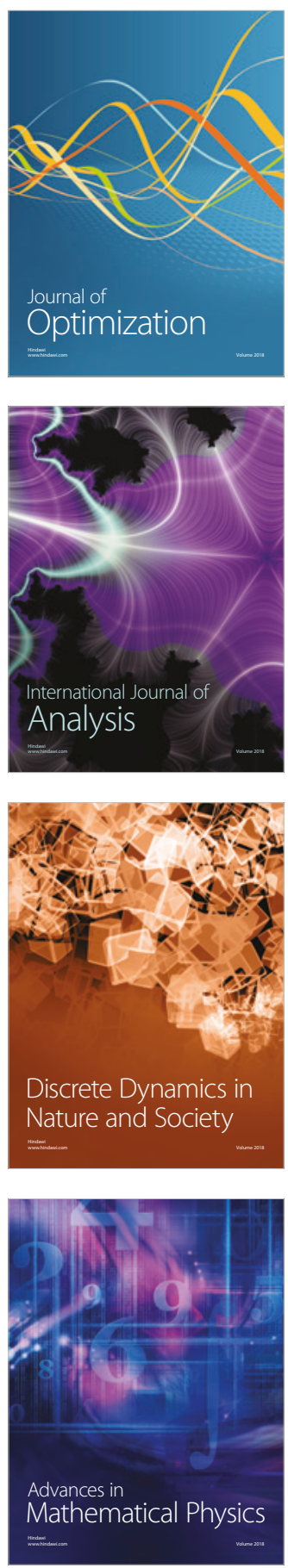